\title{
Blind spectrum sensing using symmetry property of cyclic autocorrelation function: from theory to practice
}

\author{
Lise Safatly ${ }^{1}$, Babar Aziz², Amor Nafkha2 ${ }^{2}$, Yves Louet ${ }^{2}$, Youssef Nasser ${ }^{1}$, Ali El-Hajj ${ }^{1}$ and Karim Y Kabalan ${ }^{1}$
}

\begin{abstract}
Spectrum sensing has been identified as the key step of the cognition cycle and the most important function for the establishment of cognitive radio. In this paper, a blind cyclostationary feature detector, which is based on the symmetry property of cyclic autocorrelation function (SP-CAF), is implemented and tested using universal software radio peripheral platform and GNU Radio open-source software development toolkit. Performance of the SP-CAF is compared to the classical energy detector via various tests conducted in real scenarios where both detection algorithms are employed to blindly sense the spectrum for opportunistic access. This study shows that the blind cyclostationary feature detector outperforms the classical energy detector while guaranteeing acceptable complexity and low sensing time. Moreover, different experimental results indicate that the blind sensing detector can achieve high detection probability at a low false alarm probability under real channel conditions and low signal-to-noise ratio.
\end{abstract}

\section{Introduction}

Traffic in wireless networks tends to be bursty since licensed spectrum remains unused both in time and in frequency. Hence, efficient utilization of the spectrum resources requires an opportunistic access to the unused licensed bands, commonly referred to as white spaces. Cognitive radio (CR) was initially proposed by J. Mitola and has attracted much attention due to its capability of exploring and exploiting white spaces and improving utilization of the radio spectrum [1,2]. These opportunistic capabilities of CR transceivers could be implemented under two approaches: the overlay and the underlay approaches [3]. In underlay CR, unlicensed or secondary users (SU) can transmit in any band, even the ones used by licensed or primary users (PU), but at very low power. This approach is best implemented using the ultra-wideband (UWB) technology $[4,5]$. In overlay CR, SUs are only allowed to temporarily access unoccupied spectrum allocated to primary users without causing degradation of quality of service upon the license holders. Thus, spectrum sensing is the key step in the realization of such

*Correspondence: amor.nafkha@supelec.fr

2SCEE/IETR, SUPELEC, 35576, Cesson-Sévigné Cedex, France

Full list of author information is available at the end of the article scenarios. The main goal of spectrum sensing is to accurately detect the presence or absence of a primary user in a given spectral band, under the constraint of low signal-to-noise ratio (SNR) and real channel conditions.

Recently, a new blind spectrum sensing technique based on the sparse property of the cyclic autocorrelation function (CAF) was developed for sensing the spectrum holes in the primary users' bands. This algorithm, developed lately by our research group [6], offers a suitable balance between efficiency and complexity. The main idea of this algorithm is based on using compressed sensing tools in which one could reconstruct an accurate estimation of the CAF of the received signal, and then, if this estimated function presents a symmetry (resp. no symmetry) feature, a decision on the presence (resp. absence) of the primary user could be made. It was proved that this blind detector achieves a reliable detection decision associated to a short sensing time while maintaining reasonable computational complexity.

To validate its simulated performance and complexity, this paper presents a practical study characterized by real time measurements, a code optimization approach, and a thorough testing. Firstly, theoretical work tested with simulations is not sufficient in CR scenarios and needs to be tested under real physical limitations of the hardware. For

\section{照 Springer}

(c) 2014 Safatly et al: licensee Springer. This is an Open Access article distributed under the terms of the Creative Commons Attribution License (http://creativecommons.org/licenses/by/2.0), which permits unrestricted use, distribution, and reproduction in any medium, provided the original work is properly cited. 
that, our blind spectrum sensing detector based on the symmetry property of the CAF (SP-CAF), is implemented to exploit its high performance and low sensing time. The complexity of this algorithm, which influences its sensing time, was monitored throughout the experiments and reduced by applying code optimization techniques. A different set of parameters is chosen in each experiment to describe the algorithm sensitivity. Tests are performed using Universal Software Radio Peripheral (USRP N210, Ettus Research, Santa Clara, CA, USA) [7], which is a low-cost Software Defined Radio (SDR) transceiver platform, and the GNU Radio free software [8], which is a framework for building real-time signal processing systems.

In this paper, the performance of the SP-CAF in terms of detection and false alarm probabilities and how these probabilities are influenced by the SNR are also analyzed. Moreover, the performance of SP-CAF sensing algorithm is compared with the classical energy detector (ED) technique under several real scenarios. The experimental results highlight the improved detection efficiency of the SP-CAF algorithm while utilizing a fewer number of data samples, thus guaranteeing a low computational complexity and sensing time compared to classical cyclostationary feature detector [9]. The measurements show that the SP-CAF algorithm offers an adequate tradeoff between superior performance of existing cyclostationary feature detectors and low complexity of blind detectors.

This paper is organized as follows. Section 2 introduces the system model and describes both classical ED algorithm and the blind SP-CAF sensing method, respectively. After describing the experimental setup in Section 3, various test-bed results are demonstrated in Section 4. Concluding remarks of the presented work is given in Section 5.

\section{Spectrum sensing background}

Practically, one of the most challenging issues of a cognitive terminal is spectrum sensing. In a CR scenario, the $\mathrm{SU}$ is required to identify white spaces through direct sensing of the licensed band(s). In this case, it monitors the availability of a licensed frequency band and transmits when the band is vacant. This section discusses two spectrum sensing methods for cognitive radio to ensure reliable identification of white spaces. Furthermore, major trade-offs involved in the design and optimization of such signal processing techniques are characterized.

The spectrum sensor essentially performs a binary hypothesis test on whether or not there are primary users (PU) in a particular channel. The channel is idle under the hypothesis $H_{0}$ and busy under the hypothesis $H_{1}$. It is obvious that under $H_{0}$, the received signal is only the ambient noise, whereas it consists of the PU's signal and the ambient noise under $H_{1}$. This test could be written as

$$
\begin{array}{ll}
y(k)=w(k) & H_{0} \\
y(k)=w(k)+s(k) & H_{1}
\end{array}
$$

for $k=1, \ldots, N$ where $N$ is the number of received samples, $w(k)$ represents ambient noise, and $s(k)$ is the PU signal. It is evident that the received signal will have more energy when the channel is busy than when it is idle. False alarms (or type I errors) occur if an idle channel is detected as busy, and missed detections (or a type II error) occur when a busy channel is defined as idle. A false alarm leads to a wasted opportunity for the SU to transmit, but a missed detection leads to a collision with the PU, thus a wasted transmission for both PU and SU. A clear compromise lies between ensuring the protection of the PU and providing service to the SU. A given level of protection of PU is ensured by placing a threshold on the allowed probability of missed detection. A good detector is an algorithm that minimizes the probability of false alarm, subject to a constraint on the probability of missed detection. Thus, the performance of a detector is characterized by two parameters, the probability of detection $P_{\mathrm{D}}$ and the probability of false alarm $P_{\mathrm{FA}}$. It could be observed by drawing its receiver operating characteristic (ROC) curve. ROC is a plot of $P_{\mathrm{D}}=1-P_{\mathrm{MD}}=1-\delta$, where $P_{\mathrm{MD}}$ is the probability of missed detection, versus $\epsilon=P_{\mathrm{FA}}$ for a given SNR. Choosing different detection algorithms or sensing parameters leads to different ROCs.

Several spectrum sensing methods have been proposed [10-13], including matched filter method, feature detection based on higher-order statistics like cyclostationary detection, ED method, and some emerging methods such as eigenvalue-based sensing and wavelet-based sensing. The higher-order statistics-based spectrum sensing detection in [14] can effectively separate noise from received signals even at very low SNR regime. However, it often requires a large number of samples to obtain the accurate estimations of the relevant statistics. The ED approach has been widely studied for primary users' signal detection [15]. ED is a noncoherent detector and it is shown to have low implementation complexity $[13,16]$. In addition, the ED does not require any prior knowledge about the primary user's signal. However, its detection efficiency degrades heavily under low SNR and noise uncertainty conditions [17-19], which can restrict its efficiency for cognitive radio. Indeed there exists an SNR wall beyond which energy detection is theoretically impossible.

Regardless of the used sensing algorithm, sensing errors are inevitable due to additive noise, limited observations, and the inherent randomness of the observed data. Next, we present an overview of the ED and the blind spectrum sensing algorithm based on the detection of the cyclostationarity feature of a PU introduced in [6]. 


\subsection{Energy detector}

The ED is based on the idea that with the presence of a signal in the channel, there would be significantly more energy than if there was no signal present. This concept is applicable for any PU without knowing any of its characteristics. Energy detection simply involves the application of a threshold on the collected energy from the channel. The threshold is used to decide whether a transmission is present or not. Consequently, the block diagram of the ED, illustrated in Figure 1, shows its simple detection mechanism.

Since the detection is only based on the amount of received energy, the signal can be simply modeled as a zero-mean stationary white Gaussian process, independent of the white Gaussian noise. The spectrum sensing problem distinguishes between two mutually independent Gaussian sequences $y(k)$, under hypothesis $H_{0}$ and $H_{1}$ respectively, as defined previously in Equation 1. In this equation, $w(k)$ and $s(k)$ are zero-mean complex Gaussian random variables with variances $\sigma_{w}^{2}$ and $\sigma_{s}^{2}$, respectively. Let $y=[y(1), \ldots, y(N)]^{T}$ denote the vector of the $N$ observed samples and $\sigma_{0}^{2}=\sigma_{w}^{2}, \sigma_{1}^{2}=\sigma_{w}^{2}+\sigma_{s}^{2}$.

A simple formulation of the ED detector is [20]

$$
z=\frac{1}{N \sigma_{0}^{2}} \sum_{k=1}^{N}|y(k)|^{2} \gtrless_{H_{0}}^{H_{1}} \tau
$$

where $\tau$ is the threshold chosen with respect to a given $P_{\mathrm{FA}}$ and $P_{\mathrm{MD}}$. Decreasing the threshold increases the probability of false alarm $P_{\mathrm{FA}}$. Contrarily, a low (resp. high) threshold minimizes (resp. maximizes) the probability of missed detection $P_{\mathrm{MD}}$ and thus maximizes (resp. minimizes) the probability of detection $P_{\mathrm{D}}$. It is obvious that the choice of the threshold controls the performance and the efficiency of the ED [21].

Although simple to implement, a drawback of the ED is that its performance is highly susceptible to dramatic degradations especially in low SNR scenarios. In the presence of noise uncertainty, there exists an SNR wall for ED. An SNR wall represents an SNR value, below which reliable detection is not possible even if the sensing duration is increased indefinitely [17]. On the other hand, since different transmitters employ different signal power levels and transmission ranges, one of the major concerns of energy detection is the selection of an appropriate threshold. A threshold that may work for one transmission may not be sufficient for another. All these drawbacks motivate us to study other spectrum sensing techniques that can exploit additional signal features.

\subsection{Symmetry property of cyclic autocorrelation-based detector}

To modulate signals, transmitters couple the base band signal with sine wave carriers, pulse trains, repeating spreading, hoping sequences, or cyclic prefixes which result in built-in periodicity. Thus, a modulated signal is characterized as cyclostationary since its statistics, mean and autocorrelation, exhibit periodicity. This feature can be extracted and analyzed using Fourier analysis. In communications, this periodicity is introduced intentionally so that a receiver can estimate several parameters such as carrier phase, pulse timing, or direction of arrival [22-24]. In CR networks, this periodicity is the feature for detecting a random signal with a particular modulation type in a background of noise and other modulated signals [25].

The signal $y(t)$ is said to be cyclostationary if its autocorrelation function $r_{y y}(t, \tau)$ is periodic with period $T_{0}$ :

$$
r_{y y}(t, \tau)=r_{y y}\left(t+T_{0}, \tau\right)
$$

These periodicities are examined using the CAF

$$
R_{y y}^{\alpha}(\tau)=\lim _{T \rightarrow \infty} \frac{1}{T} \sum_{t=0}^{T-1} r_{y y}(t, \tau) \exp (-j 2 \pi \alpha t)
$$

In [6], Khalaf et al. proposed to use the sparse property of the CAF to reduce the complexity of the cyclostationarity feature detector. An estimation method that utilizes the sparsity constraint is applied to estimate the CAF. Finally, to ensure the blindness of the algorithm, a symmetry test is checked on the estimated version of the CAF.

Firstly, it is shown in Equation 4 that the cyclic autocorrelation vector $\mathbf{R}_{y y}^{\alpha}$ at a given delay $\tau_{0}$ could be estimated as a scaled version of the discrete Fourier transform (DFT) of the autocorrelation vector $\mathbf{f}_{\tau_{0}}$ :

$$
\begin{aligned}
\mathbf{f}_{\tau} & =\left[f_{\tau}(0), f_{\tau}(1), \ldots, f_{\tau}(N-1)\right]^{T} \text { where } \\
f_{\tau}(t) & =y(t) y(t+\tau) \\
\mathbf{R}_{y y}^{\alpha}\left(\tau_{0}\right) & =\frac{1}{N} \operatorname{DFT}\left(\mathbf{f}_{\tau_{0}}\right)
\end{aligned}
$$

To reduce the complexity of the calculation of the cyclic autocorrelation vector, its reconstruction over $\alpha \in$ $\left[-\alpha_{\max }, \alpha_{\max }\right]$ will be done using $n$ data samples with $n \ll$ $N$ and applying compressed sensing. The key idea of compressed sensing is to recover a sparse signal, i.e., CAF in

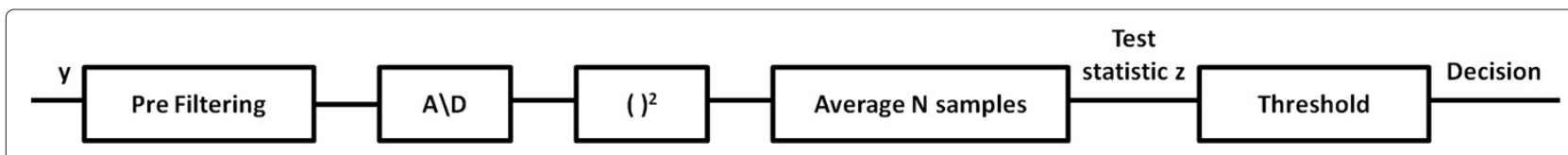

Figure 1 Block diagram of the ED. 
our case, from a small number of samples by optimization. Thus, the problem is to compute the sparse coefficient vector $\mathbf{R}_{y y}^{\alpha}$ with respect to an overcomplete system. The system representing our problem is shown as follows:

$$
A \times \mathbf{r}^{\left(\tau_{0}\right)}=\mathbf{b}^{\left(\tau_{0}\right)}
$$

with $A$ as $n$ by $N$ matrix representing the inverse discrete Fourier transform (IDFT) having the $(p, q)$ element equal to $e^{2 i \pi(p-1)(q-1) / N} . \mathbf{b}^{\left(\tau_{0}\right)}$ is an $n$-dimension vector formed by the $n$th first elements of $\mathbf{f}_{\tau_{0}}$. The solution $\mathbf{r}^{\left(\tau_{0}\right)}$ is an $N$ dimensional vector and is the solution of the problem that estimates the cyclic autocorrelation vector.

A sparse representation of such problem is

$$
\widehat{\mathbf{r}}^{\left(\tau_{0}\right)}=\min \left\|\mathbf{r}^{\left(\tau_{0}\right)}\right\|_{0} \text {, s. t. } A \times \mathbf{r}^{\left(\tau_{0}\right)}=\mathbf{b}^{\left(\tau_{0}\right)}
$$

where $\|.\|_{0}$ is the $l_{0}$-norm defined as the number of nonzero elements in the vector. To solve this sparse problem, the SP-CAF algorithm uses an iterative optimization technique called orthogonal matching pursuit (OMP) [26] that delivers an approximated solution of the following problem:

$$
\widehat{\mathbf{r}}^{\left(\tau_{0}\right)}=\min \left\|\mathbf{r}^{\left(\tau_{0}\right)}\right\|_{0} \text {, s. t. }\left\|A \times \mathbf{r}^{\left(\tau_{0}\right)}-\mathbf{b}^{\left(\tau_{0}\right)}\right\|_{2} \leq \rho
$$

OMP is based on an iterative procedure that calculates the least mean square in order to minimize the error and update residual. It uses a new atom after each iteration to guarantee a fast convergence of the algorithm. Steps of OMP are presented thoroughly in Algorithm 1.

The last step of the algorithm is to check the symmetry of the estimated cyclic autocorrelation vector. A limited number of nonzero elements are selected, and their symmetry in terms of $\alpha$ is observed. To model the symmetry test, a symmetry index $\operatorname{IND}_{\text {sym }}^{\left(\tau_{0}\right)}$ is calculated by ignoring the first amplitude that corresponds to $\alpha=0$ and by measuring the mean value of the abscissa of the remaining $l$ nonzero elements. The corresponding formula is shown as follows:

$$
\operatorname{IND}_{\mathrm{sym}}^{\left(\tau_{0}\right)}=\frac{1}{l} \sum_{i=1}^{l} \alpha_{i}
$$

It is shown that only modulated signals that have some inherited redundancy succeed in the symmetry test having $\mathrm{IND}_{\text {sym }}^{\left(\tau_{0}\right)}=0$. To achieve an efficient decision, the OMP algorithm is repeated for several values of $\tau$ and then an equivalent index is used to make the final decision. The equivalent index, shown below, is calculated by making a soft decision that combines all the indices:

$$
\mathrm{IND}_{\mathrm{sym}}^{(\mathrm{equ})}=\frac{1}{M} \sum_{i=1}^{M}\left|\mathrm{IND}_{\text {sym }}^{\left(\tau_{i}\right)}\right|
$$

To control the probability of false alarm and missed detection, a positive threshold $\xi$ is used to decide between $\overline{\text { Algorithm } 1 \text { Symmetry property of cyclic autocorrelation }}$ function detector (SP-CAF)

\section{Require:}

Initialize: Acquire $n$ data samples from the spectrum sensing interval formed by $N$ samples and set the number of OMP iterations $(l+1)$, the number $(M)$ of delays $(\tau)$, and the threshold $(\xi)$.

For $M$ different values of $\tau$,

a.) Calculate the autocorrelation vector $\mathbf{f}_{\tau_{0}}$ given by Equation 5 .

b.) Calculate the elements of the matrix $A$ performing the IDFT transform.

\section{The OMP algorithm}

c.) Initialize the residual vector of the OMP algorithm: $\operatorname{res}_{0}=\mathbf{f}_{\tau_{0}}$, the time $t=0$, and the set of indexes of the selected columns of $A$ after each iteration: $\mathrm{V}_{0}=\phi$.

d.) For $l+1$ iterations

1.) Let $v_{t}=i$, where $a_{i}$ gives the solution of $<r_{t}, a_{t}>$, where $a_{k}$ are the row vectors of $A$.

2.) Update the set $V_{t}$ with $v_{t}: V_{t}=V_{t-1} \cup$ $\left\{v_{t}\right\}$.

3.) Solve the least-squares problem: min $\left\|\mathbf{b}-\sum_{j=1}^{t} c\left(v_{j}\right) a_{v_{j}}\right\|_{2}$

4.) Calculate the new residual using $c$ : $\operatorname{res}_{t}=$ $\operatorname{res}_{t-1}-\sum_{j=1}^{t} c\left(v_{j}\right) a_{v_{j}}$

\section{End For}

\section{Symmetry check}

e.) Calculate the symmetry index $\operatorname{IND}_{\text {sym }}^{\left(\tau_{0}\right)}$ from Equation 10.

\section{End For}

Equivalent symmetry check : $\operatorname{IND}_{\text {sym }}^{(\text {equ })}=\frac{1}{M} \sum_{i=1}^{M}$ $\left|\mathrm{IND}_{\mathrm{sym}}^{\left(\tau_{i}\right)}\right|<\xi$.

hypothesis $H_{0}$ or $H_{1}$, by applying

$$
\operatorname{IND}_{\text {sym }}^{(\mathrm{equ})}<\xi
$$

The detailed version is given in Algorithm 1.

\section{Experimental implementation and code optimization}

In this section, we present the main components of our test-bed. The test-bed can be divided into two main parts, i.e., software and hardware parts. The hardware part is in charge of the RF and sampling processing while the radio transceiver's physical layer is software driven, running on a computer. 


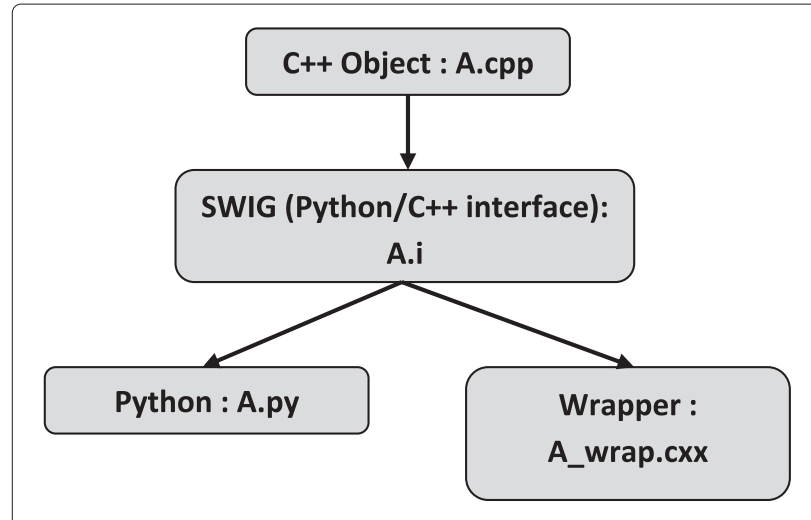

Figure 2 Interface through SWIG.

In this paper, our software part comprises GNU Radio to implement both ED and SP-CAF spectrum sensing algorithms. The GNU Radio is an open-source software toolkit for software-defined radio. This toolkit provides a number of radio components, prewritten in Python or $\mathrm{C}++$ programming languages, which can communicate to each other using various data types. Moreover, GNU Radio offers a simple and sophisticated graphical design environment, known as GNU Radio Companion (GRC), which enables users to create signal flow graphs and automatically generates Python source code. Designers can write their own signal processing blocks if needed and integrate them in GRC. Python is an object-oriented scripting language that runs on Linux/Windows and has great support for interfacing with $\mathrm{C}++$ code. This feature is often used in GNU Radio, where the signal processing blocks are written in $\mathrm{C}++$, and Python is used to 'glue' them together and control the digital data flow. This is done using Simplified Wrapper and Interface Generator (SWIG) by creating shared libraries common for both Python and $\mathrm{C}++$. In other words, SWIG is an interface compiler that connects programs written in $\mathrm{C}++$ with Python as shown in Figure 2. GNU Radio can work as a simulation environment, but it can also create a real radio system using SDR platforms. A snapshot of the receiver model designed in GNU radio is shown in Figure 3.

For the hardware part, we use a USRP N210 motherboard as the radio-frequency interface with a WBX

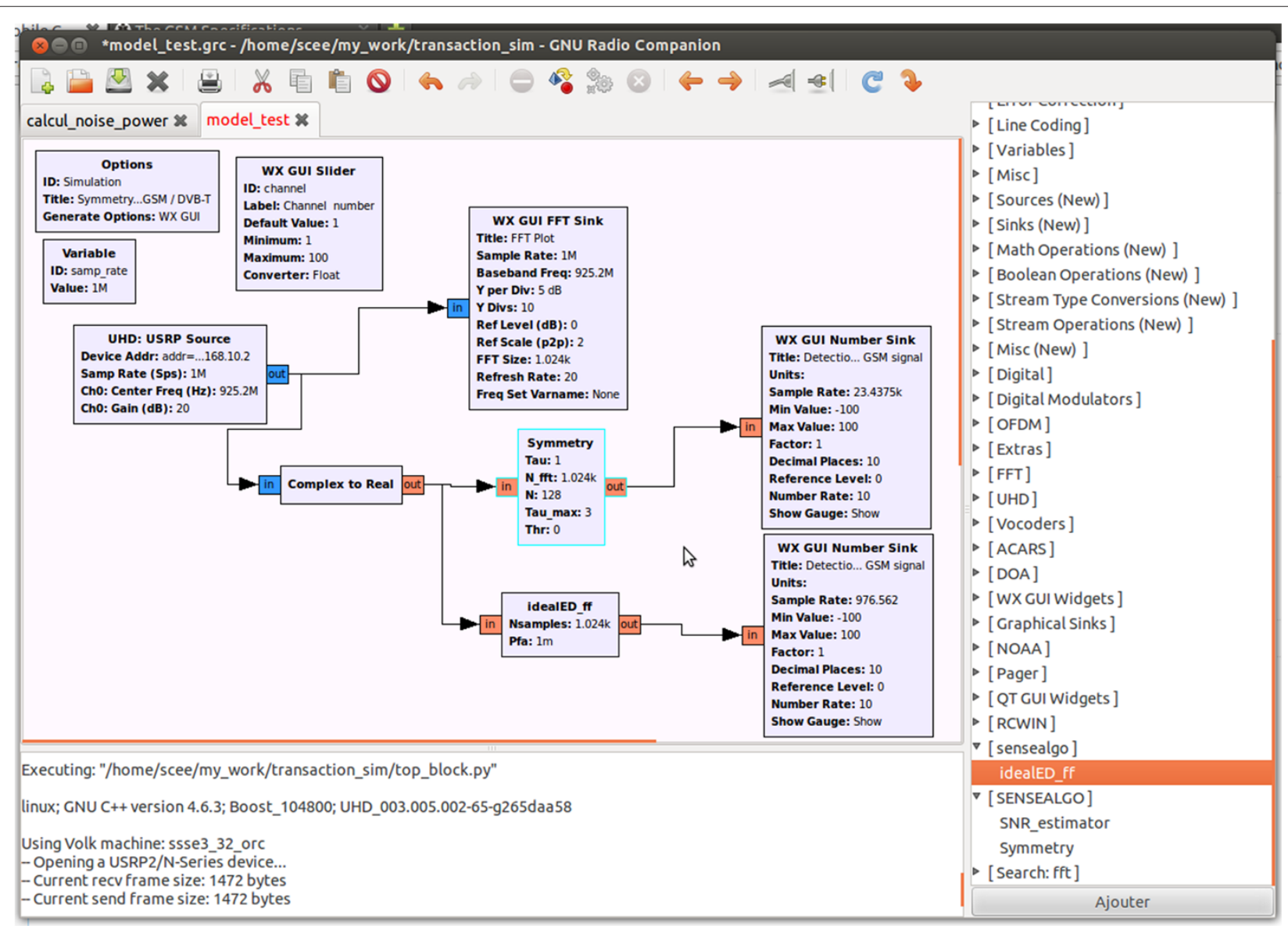

Figure 3 A snapshot of the GNU radio receiver model with ED and SP-CAF detector blocks. 


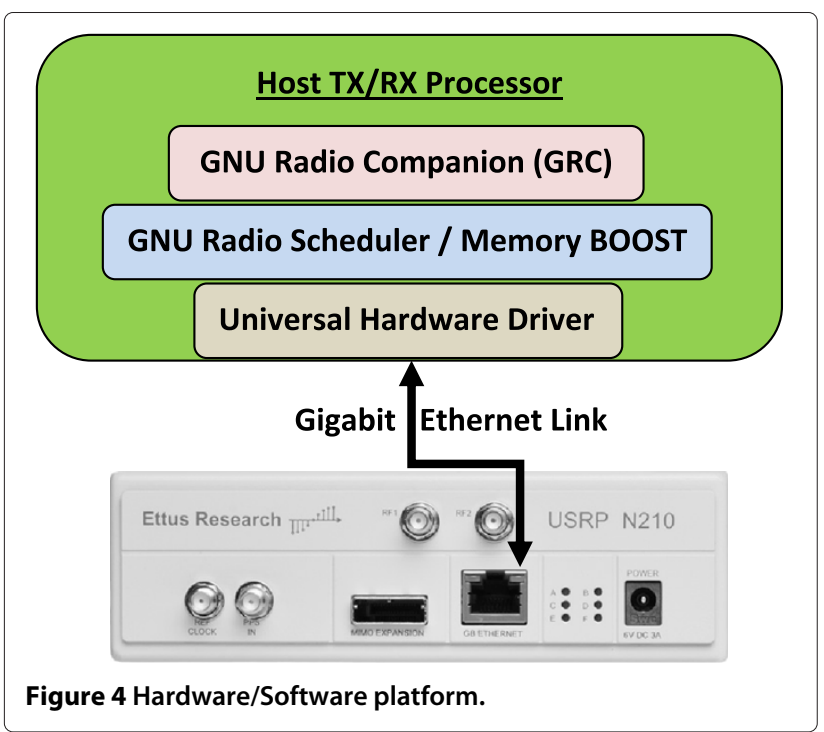

daughterboard (50- to 2,200- $\mathrm{MHz}$ receiver) and a wide band antenna to perform spectrum sensing tests. On host processors, where all signal processing blocks take place, the GRC or GNU Radio use universal hardware drivers (UHD) to send and receive signals to/from the USRP N210 board via Gigabit Ethernet link as shown in Figure 4. Figure 5 shows a snapshot of the implemented test-bed.

To minimize the complexity of this algorithm, code optimization is applied on the $\mathrm{C}++$ code level. The main complex node of the code was the generation of the IDFT matrix $A$ (see Equation 7), as concluded from the complexity tracking tool in GNU Radio. For this reason, the IDFT matrix is created off-line by creating lookup tables which are stored before running the experiments. On the other hand, several optimization techniques were applied on $\mathrm{C}++$ code such as dead store elimination, loop fusion, loop nest optimization, and register allocation. These modifications provided fast decisions of the detector.

\section{Experimental results}

In the next sections, we present some experimental results conducted at our Labs. The tests were performed on locally generated signals (Scenario 1) as well as on real standard signals captured using external antennas (Scenario 2). Firstly, measurements were recorded to estimate the SNR of the test-bed environment, and then the two scenarios are implemented to compare the SP-CAF and the ED performances. These scenarios are explained, and their respective results are presented in the next sections. The last set of experiments focus on testing the SP-CAF detector while varying its parameters.

\subsection{SNR estimation}

To properly present the detection performance at different SNR levels, we conducted measurements to estimate SNR following these steps:

Step 1. Turn the transmitter OFF, measure the power of the received signal, and set it to noise power $\left(\sigma_{w}^{2}\right)$.

Step 2. Turn the transmitter ON, measure the power of the received signal, and set it to $\sigma_{s(A)}^{2}$ where $A$ represents the transmission power level. Repeat the measurement several times in order to obtain a better estimate.

Step 3. Once the noise power and the received power are computed, the SNR at the receiver is calculated as follows:

$$
\operatorname{SNR}_{(A) \mathrm{dB}}=10 \log 10\left(\frac{\sigma_{s(A)}^{2}-\sigma_{w}^{2}}{\sigma_{w}^{2}}\right)
$$

Unfortunately, the noise power at the receiver may vary for several reasons, such as the thermal noise of the

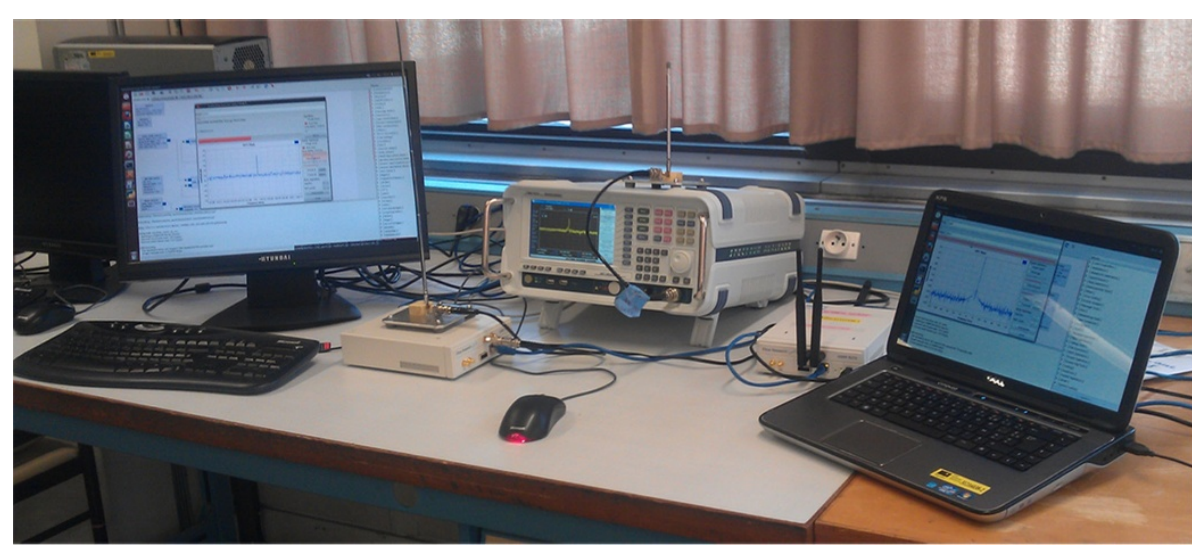

Figure 5 Snapshot of the implemented test-bed to test the performance of SP-CAF. 


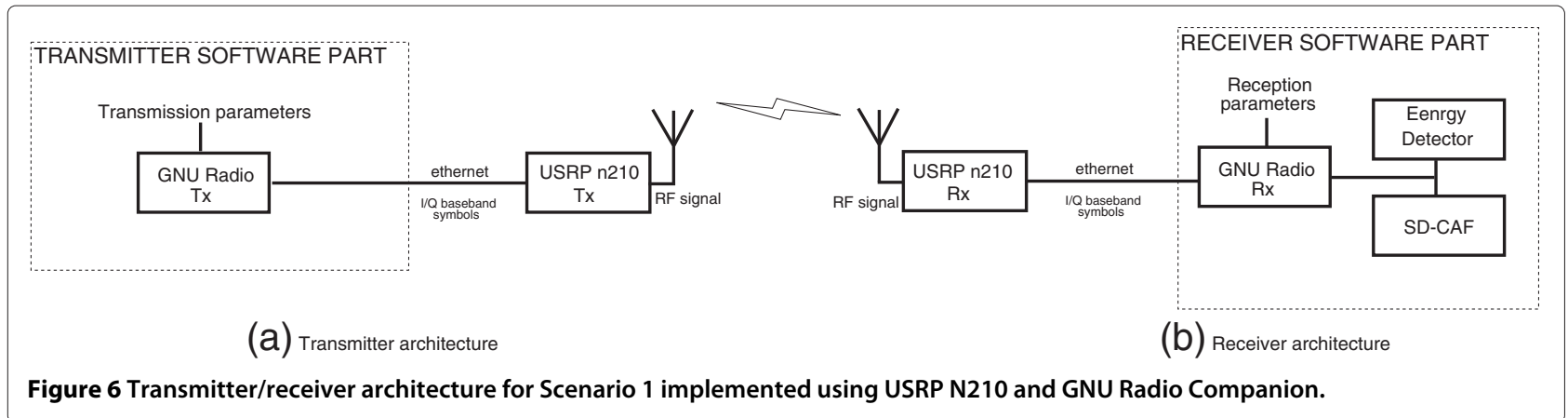

different operational stages in the receiver and another interfering transmitter in the same or adjacent frequency band. In this paper, additional care was taken to keep the received noise power as constant as possible in order to focus on the performance of the algorithm. Therefore, the measurements have been conducted in an environment where the interference has been limited, and the carrier frequency and the receiver gain were kept constant.

\subsection{Scenario 1}

The transmitter/receiver architecture for Scenario 1 is shown in Figure 6.

In this scenario, the transmitter is the primary user transmitting over an allocated band while the receiver acts as a secondary user sensing this licensed band. Firstly, an 8PSK signal is transmitted, and the receiver performs sensing via two different methods: the classical ED and the SP-CAF detector. In the experiments, the carrier frequency is set to $256 \mathrm{MHz}$. We compute the detection probability $\left(P_{\mathrm{D}}\right)$ for the two detectors at different values of the estimated SNR.

Figure 7 shows the detection probability of the two detectors obtained through the experiments. As concluded from the measurements, the probability of false alarm $\left(P_{\mathrm{FA}}\right)$ is approximately equal to 0.0078 for both detectors. It is clear from Figure 7 that the performance of the SP-CAF is better than the ED especially for SNR values less than $-12 \mathrm{~dB}$. Another important point to note is that the SP-CAF uses far less samples to perform detection compared to the ED. The number of received samples used by ED to perform detection is 16,000 compared to just 768 samples in the case of SP-CAF.

Next, in Figure 8, the detection probability of the two detectors for a sinusoid signal is plotted while maintaining the same measurement parameters. Again, it is clear also

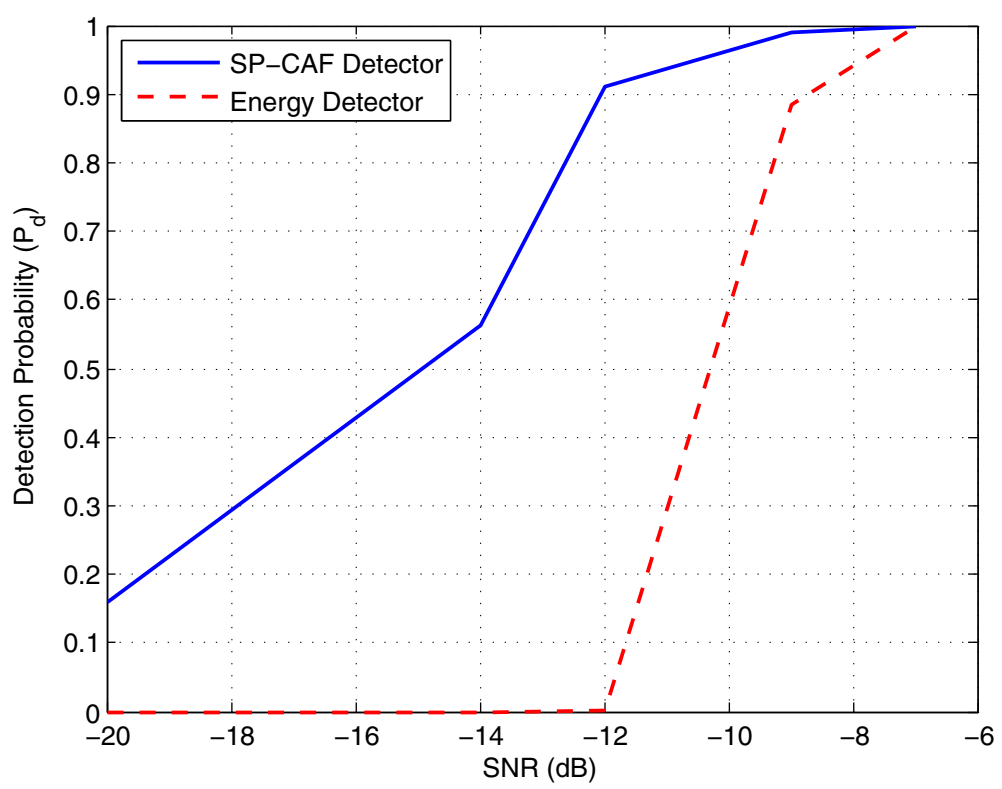

Figure 7 Probability of detection for SP-CAF vs. ED for 8PSK signal. 


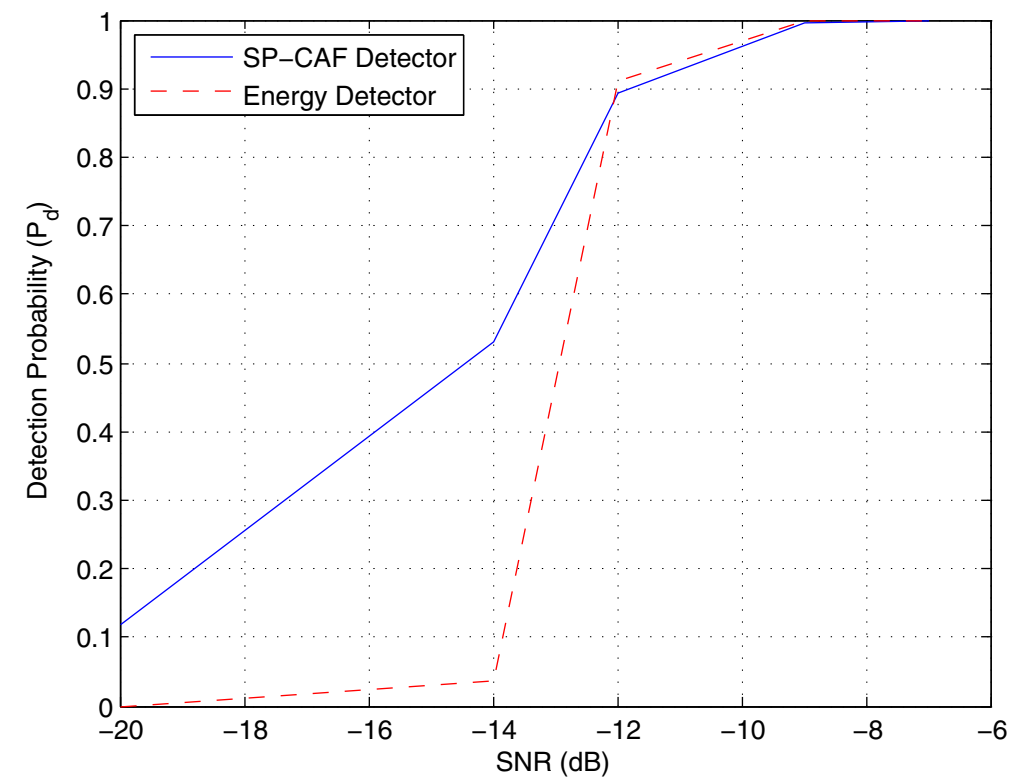

Figure 8 Probability of detection for SP-CAF vs. ED for sinusoidal signal.

that the SP-CAF outperforms the classical ED while using dramatically fewer numbers of data samples.

\subsection{Scenario 2}

Unlike the previous section where detection performance of the SP-CAF is analyzed for locally generated signals, in this section, the performance of SP-CAF is tested for real signals. Global System for Mobile Communications (GSM) and Digital Video Broadcasting-Terrestrial (DVB-T) standards were selected for the experimental tests. In the present scenario, there is only a receiver comprising one USRP N210. The experimental model for Scenario 2 is shown in Figure 9.

Firstly, the GSM band is sensed. In France, the 921- to 959-MHz band is allocated for downlink GSM transmission. Consequently, the carrier frequency of the receiver is set to $925.2 \mathrm{MHz}$. Figure 10 shows that the detection probability of the SP-CAF detector for GSM signal is equal to 1 in the sensed band.

It can be seen that the SP-CAF efficiently detects the presence of the signal as is shown by the fast Fourier transform (FFT) plot in Figure 10. Next, we perform sensing for DVB-T signals in the ultrahigh-frequency (UHF) band, while the receiver's carrier frequency is adjusted over the UHF band according to $306.188 \mathrm{MHz}+$ (Channel number) $\times 8 \mathrm{MHz}$ [27]. Note that in France, the channel number varies from 21 to 69 , and each DVB$\mathrm{T}$ channel has a bandwidth of $8 \mathrm{MHz}$. Figure 11 shows that the SP-CAF detector successfully detects the signal present in channel number 21 .

The results presented in Figures 10 and 11 highlight the performance of SP-CAF detector in terms of detection probability for real signals. On the other hand, to efficiently compare different detectors, the $\left(P_{\mathrm{FA}}\right)$ should

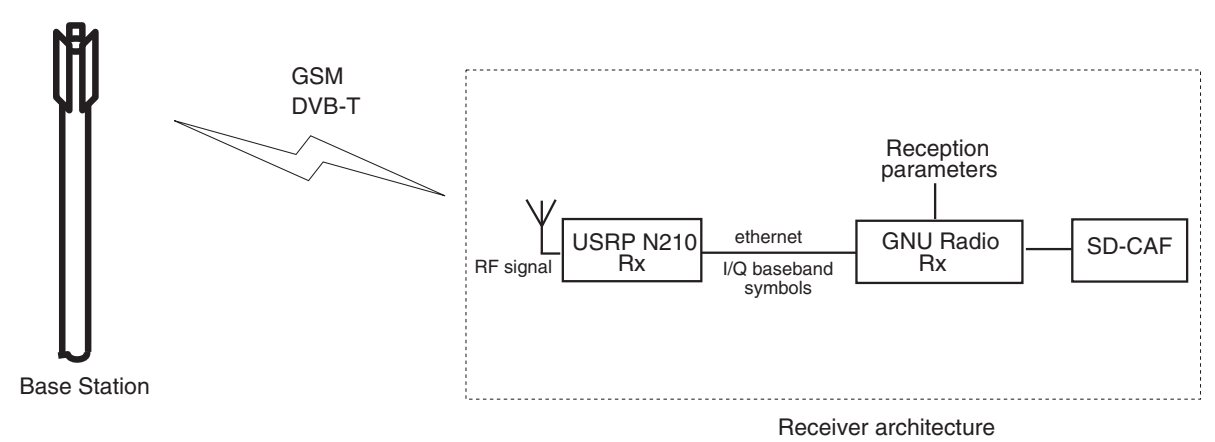

Figure 9 Transmitter/receiver architecture for Scenario 2. The receiver is implemented using USRP N210 and GNU Radio Companion. 


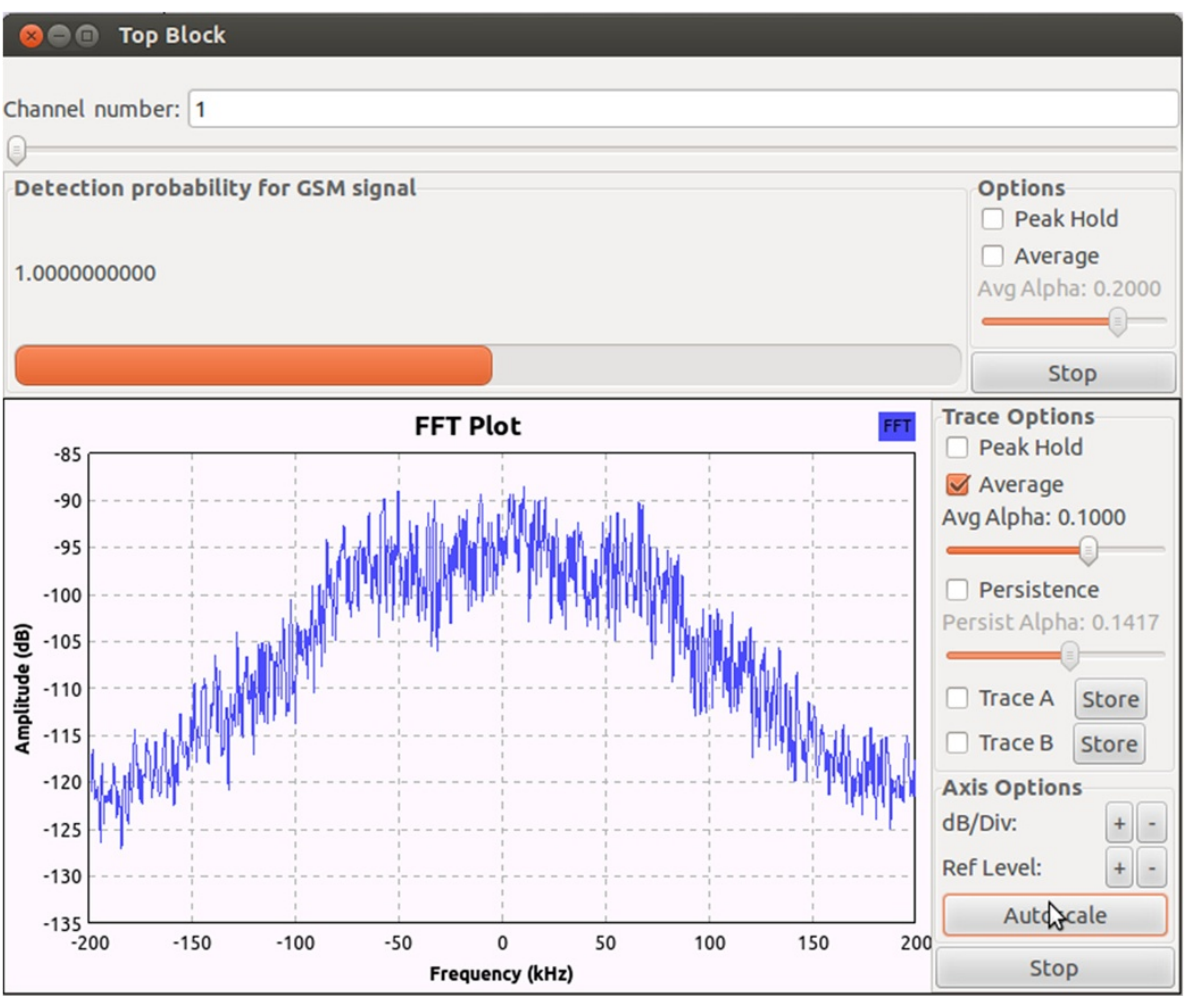

Figure 10 SP-CAF performance for real GSM signals captured with USRP N210.

$\otimes \Theta($ Top Block

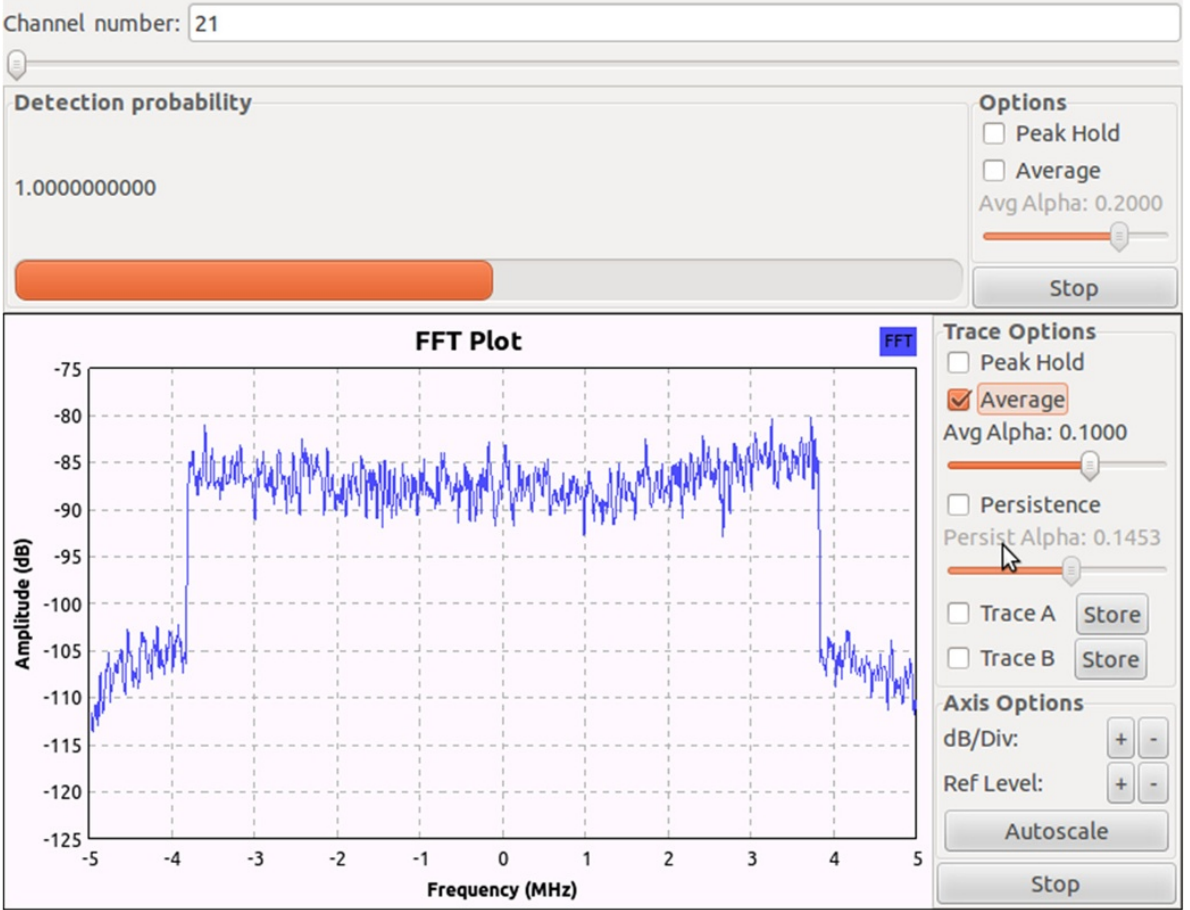

Figure 11 SP-CAF performance for real DVB-T signals captured with USRP N210. 


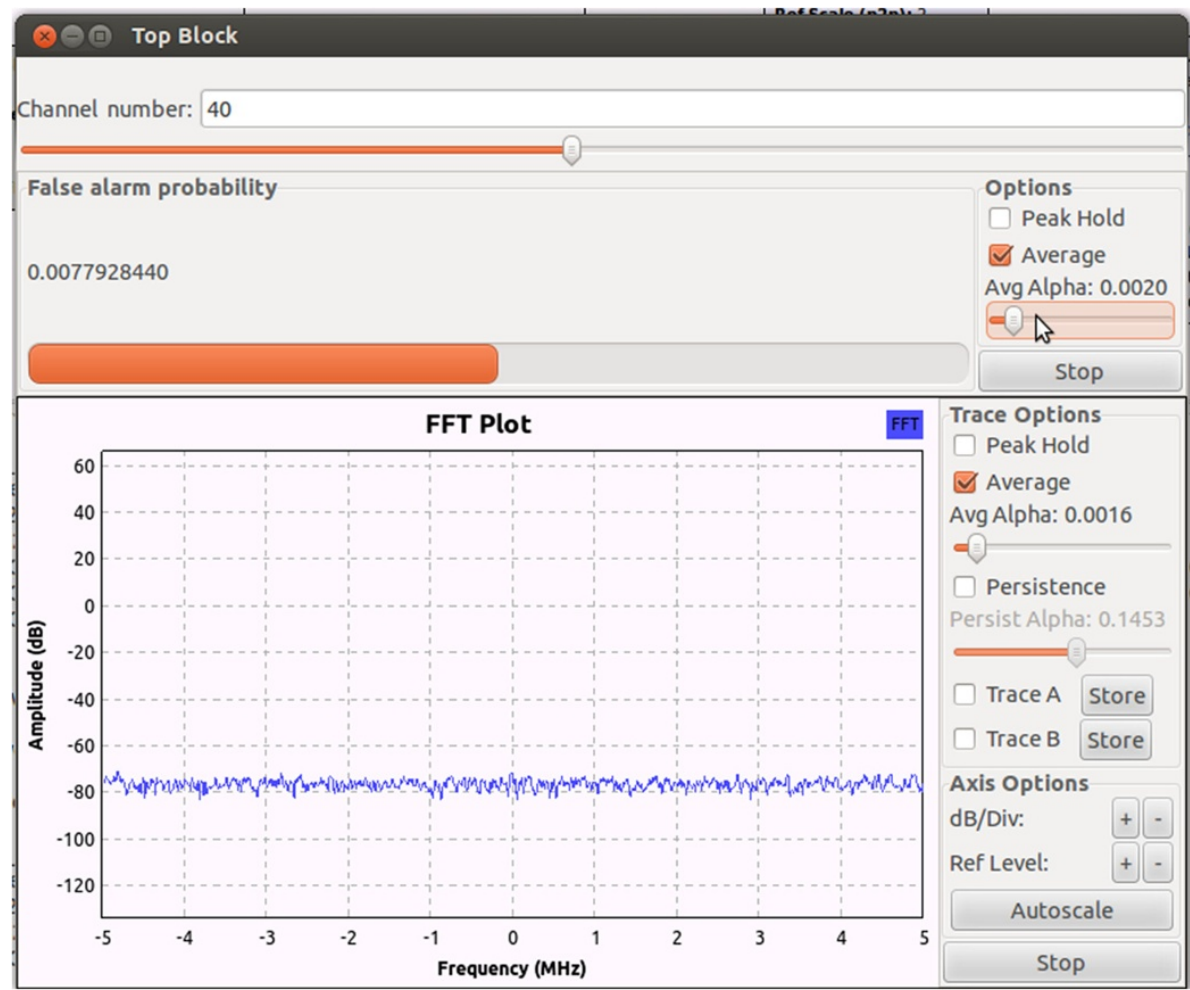

Figure 12 Probability of false detection of the SP-CAF detector.

be considered. In order to compute the $P_{\mathrm{FA}}$ for SP-CAF detector, we perform the sensing of DVB-T signal in channel number 40 since it has no DVB-T signal. However, Figure 12 shows that the SP-CAF detects a DVB-T signal with an approximate probability $P_{\mathrm{FA}}=0.0078$.
It is very important to note that the probability of false detection for the SP-CAF is very small compared to the DVB-T signals detectors presented in [28]. From the above-listed experimental results, it can be concluded that the performance of the SP-CAF detector is efficient for

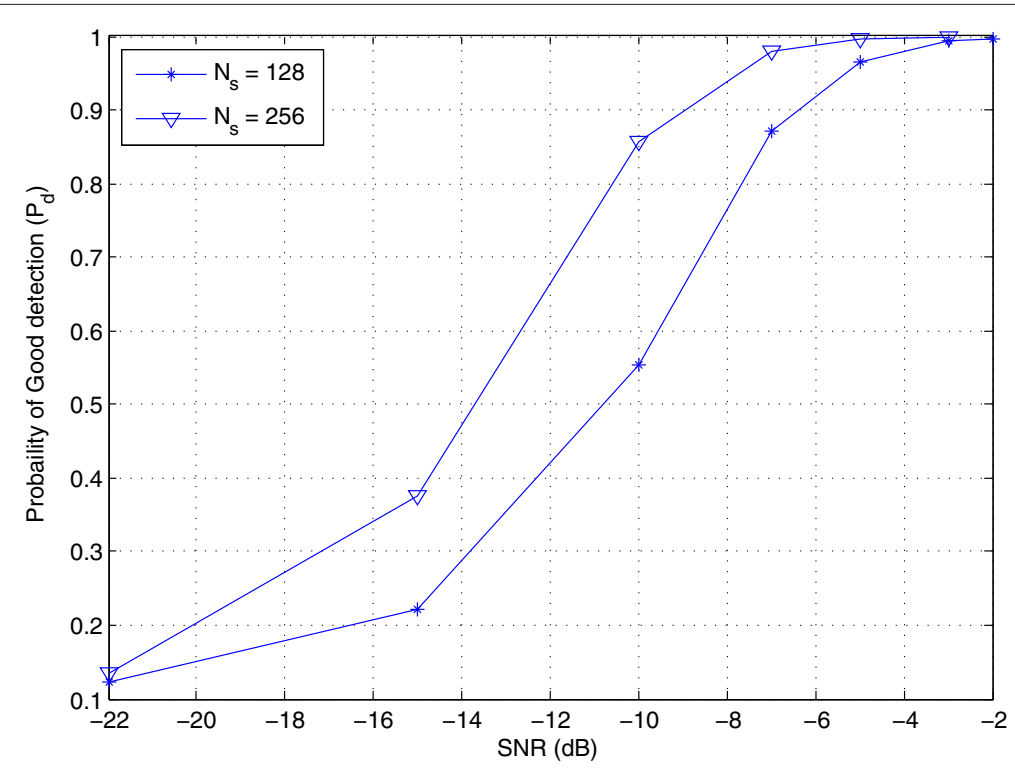

Figure 13 Effect of number of received samples on detection performance of SP-CAF. 


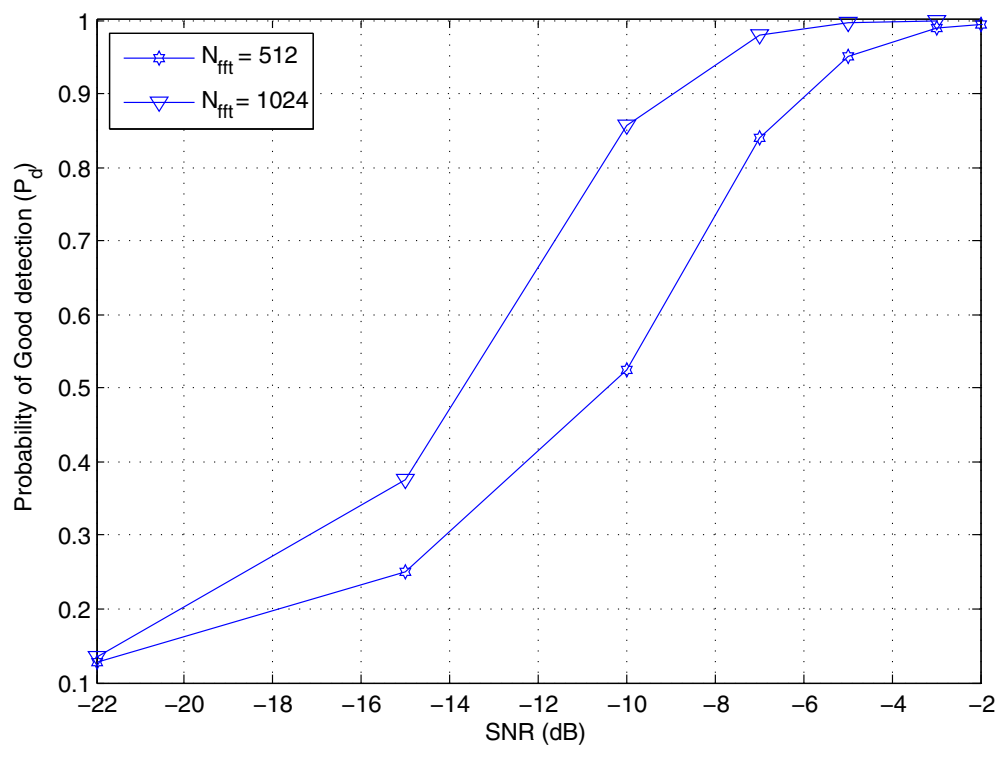

Figure 14 Effect of FFT length on the detection performance of SP-CAF.

real signals, confirmed by its high detection probability and low probability of false alarm.

\subsection{Detection performance of SP-CAF in different scenarios}

In this section, we only focus on the SP-CAF detector performance by showing the impact of its different parameters. Mainly, three parameters have an important effect on our detector's efficiency, namely
- The number of received samples used for detection $(n)$,

- The FFT length $\left(N_{\mathrm{fft}}=N\right)$,

- The maximum value of the delay $\tau$ (Equation 3) denoted as $\tau_{\max }$.

In these test scenarios, the transmitted signal is sinusoidal, and different parameters are changed successively. The measured probability of false alarm $\left(P_{\mathrm{FA}}\right)$ is equal to 0.119 . First, increasing the number of received samples

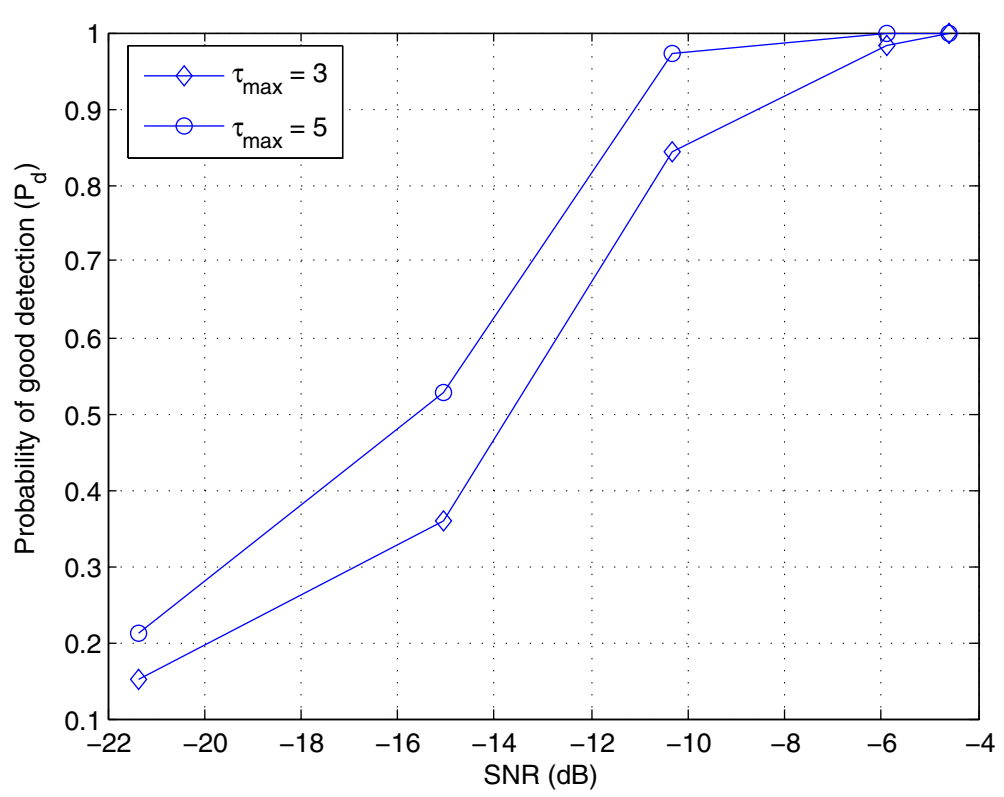

Figure 15 Effect of maximum value delay $\tau$ on detection performance of SP-CAF. 
(n) leads to a better detection probability, as shown in Figure 13. In this test scenario, the FFT length is $N_{\mathrm{fft}}=$ 1,024 and $\tau_{\max }$ is set equal to 3 . However, the curves are plotted for $n=128$ and $n=256$.

This behavior is quite obvious and self-explanatory since increasing the number of samples requires more sensing time and provides more information; thus, a better performance is obtained. In Figure 14, the detection probability $P_{\mathrm{D}}$ is plotted for different FFT lengths, i.e., $N_{\text {fft }}=512$ and 1,024 . In this case, $n$ is constant and is equal to 256 , and $\tau_{\max }$ remains equal to 3 . This figure shows that the detection probability ameliorates with the increased FFT length. As mentioned in Section 2.2, the size of the IDFT matrix is $n \times N_{\mathrm{fft}}$. Therefore, increasing $N_{\mathrm{fft}}$ increases the resolution of the estimated cyclic autocorrelation vector whose symmetry will be checked. Consequently, the detection of the symmetry property of the cyclic autocorrelation function is improved with a more accurate estimation of the CAF.

Finally, in Figure 15, the impact of $\tau_{\max }$ on the SPCAF performance is shown. In this case, $n=256$ and $N_{\mathrm{fft}}=1,024$. The probability of detection is plotted for $\tau_{\max }=3$ and 5. For $\tau_{\max }=3$, the cyclic autocorrelation vector is estimated with three values of delay $\tau$, whereas for $\tau_{\max }=5$, the estimation is performed with five values of delay $\tau$. While $\tau_{\max }$ is increasing, the estimation of the cyclic autocorrelation vector improves with a higher number of iterations, which results in a better decision on its symmetry. This is shown in Figure 15, where the detection performance of SP-CAF detector improves with a bigger $\tau_{\max }$.

\section{Conclusions}

In this paper, the performance of a blind detector that employs the symmetry property of the cyclic autocorrelation function to perform signal detection in real channel conditions is tested. The algorithm is based on the application of compressed sensing to recover the sparse CAF in the cyclic frequency domain. In this study, we have mainly focused on the practical implementation of the proposed symmetry property of SP-CAF sensing algorithm. Experimental tests were driven to compare it with the classical ED. The implementation is carried out using GNU Radio and USRP N210 platforms. It has been shown through experimental results that the SPCAF does not only require fewer samples than the classical ED but also proves to be more efficient. Moreover, the SP-CAF sensing algorithm is tested and validated on real GSM and DVB-T signals and under various test scenarios.

Competing interests

The authors declare that they have no competing interests.

\section{Acknowledgements}

This work was supported in part by Région Bretagne under grants SoftRF project and the European Commission in the framework of the FP7 Network of Excellence in Wireless COMmunications NEWCOM\# (FP7 contract number: 318306). It has been also supported by the Lebanese National Council for Scientific Research.

\section{Author details}

1 Department of Electrical and Computer Engineering, American University of Beirut, P.O. Box 11-0236, Beirut 1107 2020, Lebanon. ${ }^{2}$ SCEE/IETR, SUPELEC,

35576, Cesson-Sévigné Cedex, France.

Received: 15 July 2013 Accepted: 29 January 2014

Published: 11 February 2014

\section{References}

1. J Mitola, JGQ Maguire, Cognitive radio: making software radios more personal. Pers. Commun. IEEE. 6(4), 13 (1999). doi:10.1109/98.788210

2. S Haykin, Cognitive radio: brain-empowered wireless communications. Selected Areas Commun. IEEE J. 23(2), 201 (2005) doi:10.1109/JSAC.2004.839380

3. M Husseini, Antenna design for overlay and underlay cognitive radio applications. Ph.D. thesis. American University of Beirut, 2012

4. L Safatly, M Al-Husseini, A El-Hajj, K Kabalan, Advanced techniques and antenna design for pulse shaping in uwb cognitive radio. Int. J. Antennas Propagation (2012). doi:10.1155/2012/390280

5. M Al-Husseini, L Safatly, A El-Hajj, K Kabalan, C Christodoulou, Reconfigurable filter antennas for pulse adaptation in uwb cognitive radio systems. Prog. In Electromagnetics Res. B. 37, 327 (2012)

6. Z Khalaf, A Nafkha, Palicot J, Blind spectrum detector for cognitive radio using compressed sensing and symmetry property of the second order cyclic autocorrelation, in 7th International ICST Conference on Cognitive Radio Oriented Wireless Networks and Communications (CROWNCOM), Stockholm, 18-20 June 2012, pp. 291-296

7. Ettus Research, USRP N210 (2012). https://www.ettus.com/product/ details/UN210-KIT. Accessed 08 February 2014

8. GNU Project, GNU Radio (2013). http://gnuradio.org/redmine/projects/ gnuradio. Accessed 08 February 2014

9. AV Dandawate, G Giannakis, Statistical test for presence of cyclostationarity. IEEE Trans. Signal Process. 42(9), 2355 (1994)

10. E Axell, G Leus, E Larsson, H Poor, Spectrum sensing for cognitive radio: state-of-the-art and recent advances. Signal Process. Mag. IEEE. 29(3), 101-116. doi:10.1109/MSP.2012.2183771

11. Y Zeng, YC Liang, AT Hoang, R Zhang, A review on spectrum sensing for cognitive radio: challenges and solutions. EURASIP J. Adv. Signal Process. 2010, 15 (2010)

12. TYucek, H Arslan, A survey of spectrum sensing algorithms for cognitive radio applications. Commun. Surv. Tutor. 11(1), 116-130 (2009)

13. D Cabric, SM Mishra, RW Brodersen, Implementation issues in spectrum sensing for cognitive radios, in Proceedings of the Thirty-Eighth Asilomar Conference on Signals, Systems and Computers vol. 1 (IEEE, Piscataway, 2004), pp. 772-776

14. H Hsieh, HK Chang, ML Ku, Higher-order statistics based sequential spectrum sensing for cognitive radio, in 2011 11th International Conference on ITS Telecommunications (ITST), St. Petersburg, 23-25 Aug. 2011 (IEEE, Piscataway, 2011), pp. 696-701. doi:10.1109/ITST.2011.6060144

15. H Urkowitz, Energy detection of unknown deterministic signals. Proc IEEE. 55(4), 523-531 (1967). doi:10.1109/PROC.1967.5573

16. D Cabric, A Tkachenko, R. W Brodersen, Experimental study of spectrum sensing based on energy detection and network cooperation, in Proceedings of the First International Workshop on Technology and Policy for Accessing Spectrum (TAPAS '06) (ACM, New York, 2006)

17. R Tandra, A Sahai, SNR Walls for signal detection. Selected Topics Signal Process. IEEE J. 2(1), 4 (2008)

18. W Jouini, Energy detection limits under log-normal approximated noise uncertainty. Signal Process. Lett., IEEE. 18(7), 423-426 (2011)

19. S Bahamou, A Nafkha, Noise uncertainty analysis of energy detector: bounded and unbounded approximation relationship, in 21st European Signal Processing Conference 2013 (EUSIPCO 2013), Marrakech, Marrakech, 9-13 Sept 2013 
20. AM Wyglinski, M Nekovee, YT Hou, Cognitive Radio Communications and Networks (Academic Press, New York, 2010)

21. Z Ye, G Memik, J Grosspietsch, Energy detection using estimated noise variance for spectrum sensing in cognitive radio networks, in Proceedings of 2008 IEEE Wireless Communications and Networking Conference (WCNC 2008), Las Vegas, 31 March-3 April 2008 (IEEE, Piscataway, 2008), pp. 711-716

22. WA Gardner, Exploitation of spectral redundancy in cyclostationary signals. IEEE Signal Process. Mag. 8(2), 14-36 (1991)

23. WA Gardner, A Napolitano, L Paura, Cyclostationarity: half a century of research. Signal Process. 86(4), 639-697 (2006)

24. RW Heath Jr, GB Giannakis, Exploiting input cyclostationarity for blind channel identification in OFDM systems. IEEE Trans. Signal Process. 47(3), 848-856 (1999)

25. SXu, Z Zhao, J Shang, Spectrum sensing based on cyclostationarity, in IEEE Workshop on Power Electronics and Intelligent Transportation System (PEITS '08), Guangzhou, 2-3 August 2008 (IEEE, Piscataway, 2008), pp. 171-174

26. G Davis, S Mallat, M Avellaneda, Adaptive greedy approximations. Constr. Approximation. 13(1), 57-98 (1997)

27. Wikipedia, Television channel frequencies (2004). http://en.wikipedia.org/ wiki/Television_channel_frequencies. Accessed 08 February 2014

28. D Danev, E Axell, EGD Larsson, Spectrum sensing methods for detection of DVB-T signals in AWGN and fading channels, in 2010 IEEE 21st International Symposium on Personal Indoor and Mobile Radio Communications (PIMRC), Istanbul, 26-30 Sept 2010 (IEEE, Piscataway, 2010), pp. 772-776

doi:10.1186/1687-1499-2014-26

Cite this article as: Safatly et al.: Blind spectrum sensing using symmetry property of cyclic autocorrelation function: from theory to practice. EURASIP Journal on Wireless Communications and Networking 2014 2014:26.

\section{Submit your manuscript to a SpringerOpen ${ }^{\circ}$ journal and benefit from:}

- Convenient online submission

- Rigorous peer review

- Immediate publication on acceptance

- Open access: articles freely available online

- High visibility within the field

- Retaining the copyright to your article 